\title{
A second independent Tyr168Cys mutation in the tissue inhibitor of metalloproteinases-3 (TIMP3) in Sorsby's fundus dystrophy
}

\author{
Ute Felbor, Heidi Stöhr, Thomas Amann, Ulrich Schönherr, Eckart Apfelstedt-Sylla, \\ Bernhard H F Weber
}

\begin{abstract}
Sorsby's fundus dystrophy (SFD) is a rare autosomal dominant macular disorder with age of onset usually in the fourth decade. It is characterised by loss of central vision owing to subretinal neovascularisation and disciform macular degeneration. In an effort to identify the SFD gene, the disease locus was first mapped to chromosome 22q13-qter by genetic linkage analysis, the same chromosomal region as the gene encoding the tissue inhibitor of metalloproteinases-3 (TIMP3). Subsequently, two separate mutations in TIMP3 were found in affected members of two unrelated SFD pedigrees (Tyr168Cys and Ser181Cys). More recently, two additional SFD related mutations, Ser156Cys and Gly167Cys, have provided further confirmation that heterozygous mutations in TIMP3 are causally responsible for the SFD phenotype. We now report the occurrence of the Tyr168Cys mutation in an SFD patient of Austrian descent and show that this mutation found earlier in an American SFD family arose independently. The new findings add to an emerging pattern of SFD mutations which all seem to affect the C-terminal region of the mature TIMP3 protein. In addition, all known mutations cause a change of an amino acid to a cysteine residue. This suggests a critical role for the additional C-terminal free thiol group in SFD pathogenesis.

( $($ Med Genet 1996;33:233-236)
\end{abstract}

Key words: Sorsby's fundus dystrophy; mutational analysis; tissue inhibitor of metalloproteinases-3 (TIMP3).

The macular dystrophies represent a large group of distinct disorders that affect central vision. In this group, age related macular degeneration (AMD) has an estimated prevalence of almost $20 \%$ in the population over 65 years of age in developed countries. ${ }^{1}$ To facilitate the development of adequate therapeutic treatment, it is important to gain insights into the basic pathogenesis of these macular disorders. Clinical similarities in the early disease processes of some forms of macular degeneration have often been noted and are of particular interest for the understanding of the genetically complex AMD. Based on some common histopathological features, particularly at the level of Bruch's membrane, autosomal dominant Sorsby's fundus dystrophy (SFD) is thought to provide an excellent genetic model for the study of AMD. ${ }^{2}$

SFD is a fully penetrant autosomal dominant macular disorder characterised by loss of central vision from subretinal neovascularisation or atrophy of the retina and choroid. ${ }^{3}$ Early features of SFD include a delayed choriocapillary filling on fluorescein angiography ${ }^{4}$ and a deposition of confluent, lipid containing material between the basement membrane of the retinal pigment epithelium (RPE) and the inner collagenous layer of Bruch's membrane. ${ }^{2}$ These early manifestations in SFD appear very similar to findings in a large subgroup of patients with AMD. ${ }^{5}$

In an effort to determine the molecular basis of SFD, we first mapped the disease locus to chromosome 22q13-qter. ${ }^{6}$ This same region was shown to contain the gene encoding the tissue inhibitor of metalloproteinases- 3 (TIMP3), ${ }^{7}$ the most recently isolated member of a family of proteins that regulate the proteolytic activity of ECM degrading matrix metalloproteinases (MMPs). ${ }^{8}$ Subsequently, we detected two point mutations in TIMP3 in affected members of two SFD pedigrees suggesting TIMP3 as the SFD gene. ${ }^{9}$

More recently, two additional TIMP3 mutations, Ser 156Cys ${ }^{10}$ and Gly167Cys, ${ }^{11}$ have been identified in SFD patients. However, the pathophysiological consequences of these mutations are still unknown. The characterisation of additional mutations in the SFD gene and, in particular, in vitro mutagenesis is likely to provide new clues to the mechanism by which such mutations may cause the clinical features in SFD.

\section{Case report}

At the age of 33, our patient experienced a sudden loss of central vision in the right eye resulting from subretinal macular haemorrhage with glial cicatrisation of the neuroretinal layer. The visual acuity was $20 / 400$ right eye with $-3 \mathrm{sph}$ and $20 / 25$ left eye with $-4.5 \mathrm{sph}$, -0.75 cyl axis $170^{\circ}$. On funduscopy of the right eye, there was a large disciform glial scar of the macula surrounded by accumulations of pigment and atrophic zones of the RPE and choriocapillaris. In the posterior pole of the left eye, the optic disc appeared normal, but there was RPE atrophy peripheral to the fovea with 
List of oligonucleotide primer sequences used to amplify the five coding exons of TIMP3

\begin{tabular}{llllr}
\hline Exon & Primer & $\begin{array}{l}\text { Primer sequences } \\
\text { (given in the 5' to 3' orientation) }\end{array}$ & $\begin{array}{l}\text { Restriction } \\
\text { enzyme }\end{array}$ & $\begin{array}{c}\text { Fragment } \\
\text { sizes }\end{array}$ \\
\hline 1 & ex 1 & CTT TGG AGA GGC GAG CAG & PvuII & 85 \\
2 & ex 1R & TCG GGC GGG GCA CCA GGA & AluI & 109 \\
2 & ex 2 & TCG TGT TCC TGA TGT GGT T & 90 \\
& ex 2R & CGG AGC CAG GGT GAT GAC & Ha & 81 \\
3 & ex 3 & GGA AAG AAG AAG CTA TGA TG & Hin & 96 \\
& ex 3R & AGA AAC AGT GGA CCT TGG C & RsaI & 106 \\
4 & ex 4 & GAC AAA ACA ACC TCT CCT T & 65 \\
& ex 4R & TCA GCA CCT CCC CTC CTT & PstI & 139 \\
5 & 2FF & GTC CAT CAA CTG CTG CCT G & 172 \\
& ex 5R & AAG GGA GGG AAG TGA GGT & & 94 \\
\hline
\end{tabular}

regional atrophy of the choriocapillaris. Fine drusen-like deposits of the RPE layer were irregularly scattered throughout the posterior poles and the midperipheral regions bilaterally. Regions of RPE atrophy with pigment clumping could be detected in all quadrants. On static perimetry, there was an absolute central scotoma of the right eye and a relative central scotoma of the left eye, both extending to about $15^{\circ}$ peripherally. Standard electroretinography (ERG) showed normal rod and cone signals in both eyes.

At the age of 37, the patient presented with an abrupt decrease in central vision in the other eye owing to fresh choroidal neovascularisation with slight subretinal haemorrhage and serous retinal detachment. Dark adaptation was $2 \mathrm{log}$ units above normal bilaterally. Electro-oculography (EOG) showed subnormal light/dark ratios (1.54 RE and 1.58 LE). Standard scotopic ERG showed a selective rod response within normal limits whereas standard flash and photopic ERG were subnormal.

The family history remains unclear as the patient's mother and two brothers (aged 34 and 32) showed no characteristic ophthalmological features of SFD, while the father and the paternal grandmother died early in life. The father's sibs had been adopted by various families.

\section{Materials and methods}

The human TIMP3 gene consists of five coding exons distributed over approximately $55 \mathrm{~kb}$ of genomic DNA. ${ }^{12}$ PCR primer pairs flanking the exons are listed in the table. The conditions for PCR amplification have been reported elsewhere. ${ }^{910}$ Briefly, after an initial denaturation of one minute at $94^{\circ} \mathrm{C}, 30$ cycles of 30 seconds at $94^{\circ} \mathrm{C}, 30$ seconds at $55^{\circ} \mathrm{C}$ (exon 1 and 5 ) or at $52^{\circ} \mathrm{C}$ (exons 2,3 , and 4 ), and 30 seconds at $72^{\circ} \mathrm{C}$ were carried out followed by a final extension of five minutes at $72^{\circ} \mathrm{C}$.

To increase sensitivity of the single stranded conformational polymorphism (SSCP) analysis, ${ }^{13}$ the PCR products were digested with various restriction enzymes to yield fragments $<200 \mathrm{bp}$ (table). A combination of two gel running conditions was applied using $6 \%$ non-denaturing polyacrylamide gels with (5\%) and without glycerol at $4^{\circ} \mathrm{C}, 25 \mathrm{~W}$ constant power for four to seven hours.

Exon 5 PCR fragments were cloned into the pCR II vector (Invitrogen). Recombinant clones were sequenced using the dideoxy nucleotide chain termination method (USB, Cleveland) and sequencing primers $\mathrm{M} 13(-40) \mathrm{F}$ (5'GTT TTC CCA GTC ACG A-3') and M13 $(-40)$ R (5'-CAG GAA ACA GCT ATG AC$\left.3^{\prime}\right)$. Genotyping was performed using $(\mathrm{CA})_{\mathrm{n}}$ dinucleotide repeat markers at loci D22S275 and D22S281 and PCR conditions as described. ${ }^{14}$ The intragenic polymorphism in exon 3 of the TIMP3 gene is the result of a silent mutation in codon 60 and 64, respectively, and can be analysed by SSCP of exon 3 PCR fragments amplified with primers ex3/ex3R (table).

\section{Results}

To test the Austrian patient for mutations in TIMP3, we performed SSCP of the entire
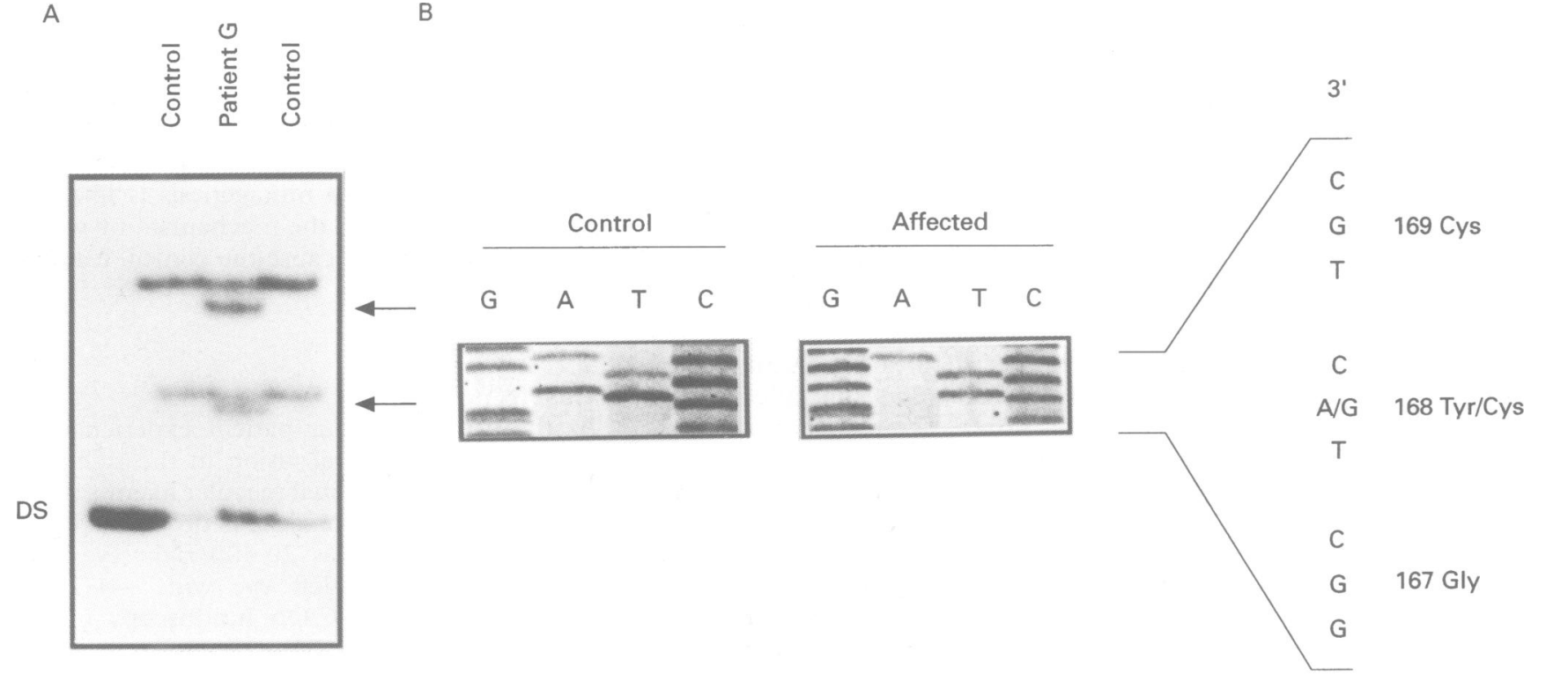

$5^{\prime}$

Figure 1 (A) A SSCP mobility shift in exon 5 of the TIMP3 gene in Austrian SFD patient G. For comparison, two controls are shown (DS=double strand). (B) Sequencing of exon 5 of TIMP3 shows a heterzygous $A$ to $G$ transition in the second base of codon 168 resulting in a Tyr168Cys alteration in the mature TIMP3 protein. 


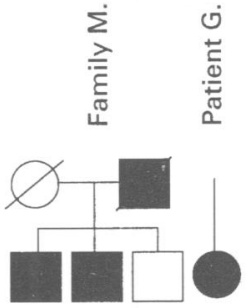

$\begin{array}{lllll}\text { D22S275 } & 23 & 34 & 23 & 11 \\ \text { TIMP3 ex3 } & 12 & 11 & 22 & 22 \\ \text { D22S281 } & 14 & 13 & 45 & 24\end{array}$

B

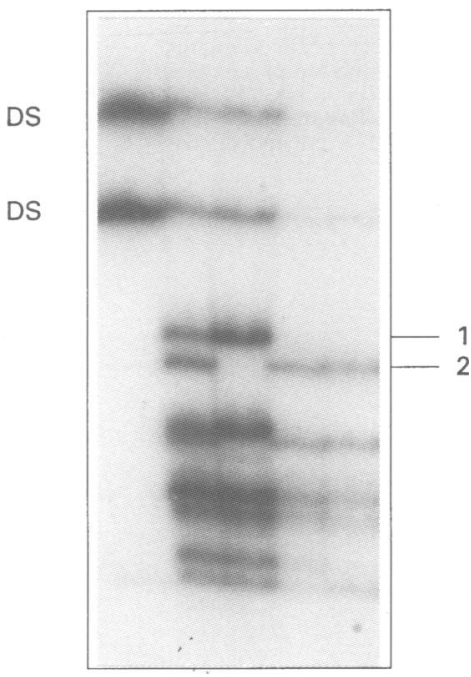

Figure 2 (A) Genotyping of the members of the American SFD family $M^{9}$ and the Austrian SFD patient $G$, with markers flanking the SFD locus as well as an intragenic TIMP3 polymorphism. The Austrian SFD patient does not share alleles associated with SFD in the American family. (B) An intragenic polymorphism in exon 3 of the TIMP3 gene shows that the Austrian SFD patient is homozygous for the lower mobility shift (2) which is not linked to the Tyr168Cys mutation in the American SFD patients.

coding sequence of the gene. Analysis of exon 5 showed a band shift (fig 1A) which was not found in her two brothers or in 270 unaffected controls. However, this mobility shift was similar to a TIMP3 mutation in exon 5 previously identified in an American SFD family. ${ }^{9}$

Sequencing of the mutant allele showed an A to $G$ transition in the second position of codon 168 changing a highly conserved tyrosine residue $^{71516}$ to a cysteine (fig $1 \mathrm{~B}$ ). These alterations are identical to the findings in the American SFD family. ${ }^{9}$

To ascertain whether the Tyr168Cys mutation in the American SFD family ${ }^{9}$ and our Austrian SFD patient originated from a common ancestor, we genotyped the critical subjects with $(\mathrm{CA})_{\mathrm{n}}$ dinucleotide repeat markers at D22S275 and D22S281 as well as a frequent intragenic SSCP in exon 3 of the TIMP3 gene. The Austrian patient does not carry the particular alleles at D22S275 and D22S281 associated with SFD in the American family (fig $2 A)$. In addition, the intragenic SSCP in exon
3 shows that the band shift segregating with SFD in the American family is not shared by the Austrian SFD patient (fig 2B).

\section{Discussion}

To date, four different mutations have been identified in TIMP3 in patients diagnosed with Sorsby's fundus dystrophy, including a Ser156 Cys mutation in a German-Czech family with an atypical early onset and a rapid progression of the disease, ${ }^{10}$ a Gly167Cys mutation, ${ }^{11}$ a Tyr 168 Cys mutation in an American family, ${ }^{9}$ and a Ser181Cys mutation in a Canadian family of Irish descent. ${ }^{9}$ In addition, we report here a second Tyr168Cys mutation in an Austrian patient and unambiguously show its independent occurrence from the identical American mutation.

Despite this relatively small number of known mutations in SFD, a mutational pattern underlying the disease appears to emerge. So far, all mutations have been identified in exon 5 of the TIMP3 gene, thus affecting the Cterminal region of the mature protein. Although little is known about the kinetics of TIMP3 action, the C-terminal domains of TIMP1 and TIMP2 are thought to increase binding stability between the inhibitor and its matrix metalloproteinases and, thus, to influence the rate of inhibition through low affinity interactions. ${ }^{178}$ In addition, the $\mathrm{N}$-terminal domains of TIMP1 and TIMP2 alone retain metalloproteinase inhibitory function and appear not to require their C-terminal domains for full activity. ${ }^{1920}$ This suggests that the mutational changes in the C-terminal domain of TIMP3 may not fully interfere with TIMP3 inhibitory function and could explain, at least in part, the late onset of symptoms usually in the fourth decade of life. ${ }^{3}$ Similarly, partial activity of the mutated TIMP3 protein could also account for the absence of pathological features in other body tissues with high TIMP3 expression. $^{715}$

It is striking that all known TIMP3 mutations lead to the introduction of an additional cysteine residue in the peptide sequence suggesting that the mechanism underlying the SFD pathology may be directly related to the presence of a free thiol group in the C-terminal part of the peptide. This raises the question of whether other types of mutations in the TIMP3 gene could be responsible for other as yet unknown clinical conditions different from SFD. The study of transgenic animals carrying various types of mutations in the TIMP3 gene may provide some clues to the effect of specific mutations on the resulting clinical phenotype.

The authors thank the patient and her family for their cooperation and Drs A Baumer and M Gessler for their helpful comments on the manuscript.

1 Klein R, Klein BE, Linton KLP. Prevalence of age-related maculopathy: the Beaver Dam Eye Study. Ophthalmology 1992;99:933-43.

2 Capon MRC, Marshall J, Krafft JI, Alexander RA, Hiscott PS, Bird AC. Sorsby's fundus dystrophy: a light and electron microscopic study. Ophthalmology 1989;96:176977 .

3 Sorsby A, Mason MEJ, Gardener N. A fundus dystrophy with unusual features. $B r \mathcal{F}$ Ophthalmol 1949;33:67-97. 
4 Polkinghorne PJ, Capon MRC, Berninger T, Lyness AL Sehmi K, Bird AC. Sorsby's fundus dystrophy: a clinical study. Ophthalmology 1989;96:1763-8.

5 Pauleikhoff D, Chen JC, Chisholm IM, Bird AC. Choroida perfusion abnormality with age-related Bruch's membran change. Am $\mathcal{f}$ Ophthalmol 1990;109:211-7.

6 Weber BHF, Vogt G, Wolz W, Ives EJ, Ewing CC. Sorsby's fundus dystrophy is genetically linked to chromosome 22q13-qter. Nature Genet 1994;7:158-61.

7 Apte SS, Mattei MG, Olsen BR. Cloning of the cDNA encoding human tissue inhibitor of metalloproteinases-3 (TIMP-3) and mapping of the TIMP3 gene to chromosome 22. Genomics 1994:19:86-90.

8 Matrisian LM. Metalloproteinases and their inhibitors in matrix remodeling. Trends Genet 1990;6:121-5.

9 Weber BHF, Vogt G, Pruett RC, Stöhr H, Felbor U. Mutations in the tissue inhibitor of metalloproteinases- 3 (TIMP3) in patients with Sorsby's fundus dystrophy. Nature Genet 1994;8:352-6.

10 Felbor U, Stöhr H, Amann T, Schönherr U, Weber BHF. A novel Ser156Cys mutation in the tissue inhibitor of metalloproteinases-3 (TIMP3) in Sorsby's fundus dystrophy with unusual clinical features. Hum Mol Genet 1995;4:2415-16.

11 Jacobson SG, Cideciyan AV, Regunath G, et al. Night blindness in Sorsby's fundus dystrophy reversed by vitamin A Nature Genet 1995;11:27-32.

12 Stöhr H, Roomp K, Felbor U, Weber BHF. Genomic organization of the human tissue inhibitor of metalloorganization of the human tissue inhibitor of
proteinases-3 (TIMP3). Genome Res (in press).

13 Orita M, Suzuki Y, Sekiya T, Hayashi K. Rapid and sensitive detection of point mutations and DNA polymorphisms using the polymerase chain reaction. Genomics 1989;5: $874-9$.

14 Weissenbach J, Gyapay G, Dib C, et al. A second-generation inkage map of the human genome. Nature 1992;359. 794-801.

15 Leco KJ, Khokha R, Pavloff N, Hawkes SP, Edwards DR. Tissue inhibitor of metalloproteinases-3 (TIMP3) is an extracellular matrix-associated protein with a distinctive pattern of expression in mouse cells and tissues. $\mathcal{F} \mathrm{Biol}$ Chem 1994;269:9352-60

16 Pavloff N, Staskus PW, Kishnani NS, Hawkes SP. A new inhibitor of metalloproteinases from chicken: ChIMP-3 A third member of the TIMP family. F Biol Chem 1992;

17 Willenbrock F, Crabbe T, Slocombe PM, et al. The activity of the tissue inhibitors of metalloproteinases is regulated by C-terminal domain interactions: a kinetic analysis of the inhibition of gelatinase A. Biochemistry 1993;32:4330-7.

18 Nguyen Q, Willenbrock F, Cockett MI, O'Shea M, Docherty AJP, Murphy G. Different domain interactions are involved in the binding of tissue inhibitors of metalloproteinases to stromelysin-1 and gelatinase A. Biochemistry 1994;33:2089-95.

19 Murphy G, Houbrechts A, Cockett MI, Williamson RA, O'Shea M, Docherty AJP. The N-terminal domain of tissue inhibitor of metalloproteinases retains metalloproteinase inhibitory activity. Biochemistry 1991;30:8097102.

20 DeClerck YA, Yean TD, Lee Y, Tomich JM, Langley KE. Characterization of the functional domain of tissue in hibitor of metalloproteinases-2 (TIMP-2), Biochem $\mathcal{F}$ 1993;289:65-9. 